COLABORACIÓN ESPECIAL

\title{
PLAN PARA LA ELIMINACIÓN DEL SARAMPIÓN Y LA RUBEOLA EN ESPAÑA
}

\section{Aurora Limia Sánchez.}

Subdirección General de Promoción de la Salud y Epidemiología. Dirección General de Salud Pública, Calidad e Innovación. Ministerio de Sanidad, Servicios Sociales e Igualdad.

La autora declara no tener conflicto de intereses.

\section{RESUMEN}

El Plan de Eliminación del Sarampión en España, del año 2001, y el Protocolo de vigilancia de la rubeola y del síndrome de la rubeola congénita en fase de eliminación, del año 2007, componen el actual Plan para la eliminación de ambas enfermedades en España. Desde 2010 se está reforzando el seguimiento de la eliminación del sarampión y la rubeola en la Región Europea de la Organización Mundial de la Salud (OMS), solicitando información anual a los estados miembros sobre la situación del sarampión y la rubeola desde el año 2013. Ese mismo año, a instancias de la OMS, el Ministerio de Sanidad, Servicios Sociales e Igualdad nombró el Comité Nacional de Verificación de la Eliminación del Sarampión y la Rubeola en España para la revisión y envío de la información solicitada.

En este artículo se comentan las estrategias y actividades actuales recomendadas por la Región Europea de la OMS, el proceso de verificación en España y los pasos a seguir para la actualización del Plan de eliminación del sarampión y la rubeola en España.

Palabras clave: Sistema de información. Sarampión. Rubéola. Vacunación masiva. Vacuna contra el sarampión.

Correspondencia

Aurora Limia Sánchez

alimia@msssi.es

DOI:

\section{ABSTRACT \\ Plan for the Elimination of Measles and Rubella in Spain}

The current Plan for the elimination of measles and rubella is made of the Plan for the elimination of measles in Spain, set up in 2001, and the Surveillance protocol of rubella and congenital rubella syndrome in the phase of elimination, established in 2007. The monitoring of the elimination of both diseases conducted from the European Region of World Health Organization (WHO) has been reinforced since 2010. An annual report with information on the situation of measles and rubella in The Member Estates is being requested since 2013. The Ministry of Health, Social Services and Equality nominated a National Committee for Verification of Measles and Rubella Elimination in 2013, for the review and delivery of the requested information.

The strategies and activities currently recommended from the WHO European Region, the verification process in Spain, and the steps to follow for the updating of the Plan for the elimination of measles and rubella in Spain are mentioned in this article.

Keywords: Information system. Measles. Rubella.Vaccines. Vaccination. Mass vaccination. Measles vaccine. 


\section{ANTECEDENTES}

El "Plan de eliminación del sarampión en España" se estableció en 2001 en base a la aprobación por el Comité de la Región Europea de la OMS, en 1998, del marco de políticas Salud para todos en el siglo 21. En este documento de la OMS se identificaban metas para nueve enfermedades prevenibles mediante vacunación, incluyendo la eliminación del sarampión para el año 2007 y la reducción de la incidencia de síndrome de rubeola congénita a menos de un caso por 100.000 nacidos vivos en 2010 . Posteriormente, en 2004, el grupo asesor de vacunación de la Región, European Technical Advisory Group of Experts on Immunization (ETAGE), recomendó la inclusión de la rubeola en la estrategia de eliminación, que se aprobó en la $55^{\mathrm{a}}$ sesión del Comité de la Región Europea de la OMS en 2005 y quedó plasmado en el Plan estratégico 20052010 .

Para cumplir con el objetivo de reforzar la vigilancia en España, se elaboró el "Protocolo de vigilancia de la rubéola y del síndrome de rubéola congénita en la fase de eliminación", que se aprobó por la Comisión de Salud Pública en julio de 2008 como ampliación del Plan de eliminación del sarampión .

Con posterioridad, en la $60^{\mathrm{a}}$ sesión del Comité Regional en 2010, tras revisar los progresos realizados en la prevención de estas enfermedades en los países de la Región, se amplió la meta para la eliminación al año 2015, y se estableció el proceso de verificación de la eliminación como apoyo para su consecución ${ }^{4}$.

En este artículo se revisan las estrategias y actividades fundamentales coordinadas desde la Región Europea de la OMS para la eliminación, el proceso de verificación y se comentan los pasos a seguir para la actualización del Plan de eliminación de sarampión y rubeola en España.

\section{ESTRATEGIAS Y ACTIVIDADES PARA LA ELIMINACIÓN}

Para detener la circulación del virus de sarampión, tanto endémico como importado, se deben dirigir todos los esfuerzos a alcanzar altas coberturas de vacunación sistemática y una amplia inmunidad comunitaria en todos los países. La menor infectividad del virus de la rubeola y la mayor protección conferida por la vacuna, incluso con una sola dosis, puede hacer que su eliminación suponga un menor reto y que los esfuerzos para conseguir la eliminación del sarampión puedan aprovecharse para la eliminación adicional de la rubeola.

Las estrategias fundamentales establecidas por la Región Europea de la OMS para la eliminación incluyen ${ }^{4,8}$ :

1. Alcanzar y mantener altas coberturas de vacunación $(\geq 95 \%)$ con dos dosis frente a sarampión y al menos una dosis frente a rubeola mediante vacunación sistemática.

2. Proporcionar oportunidades de vacunación para todos los grupos de riesgo y otros grupos susceptibles, las cuales pueden incluir actividades de vacunación complementaria.

3. Fortalecer los sistemas de vigilancia realizando la investigación rigurosa de cada caso detectado y la confirmación de laboratorio de todos los casos esporádicos y los brotes.

4. Mejorar la disponibilidad de información de alta calidad y basada en la evidencia científica para todos los profesionales y la población sobre los beneficios y el riesgo asociado de la vacunación frente a sarampión y rubeola.

5. Verificar la eliminación de sarampión y rubeola.

Para el establecimiento de las estrategias se recomienda establecer actividades centradas en seis categorías ${ }^{8}$ que incluyen: 1) fortalecimiento en el sistema de vacunación, 2) fortalecimiento del sistema de vigilancia epidemiológica, 3) prevención de brotes y pre- 
paración de la respuesta a los mismos, 4) comunicación, información y apoyo, 5) movilización de recursos y asociaciones y 6) verificación de la eliminación de sarampión y rubeola.

\section{PROCESO DE VERIFICACIÓN DE LA ELIMINACIÓN}

En enero de 2012 se mantuvo la primera reunión de la Comisión Regional de Verificación para la Eliminación del Sarampión y la Rubeola, Regional Verification Commission for Measles and Rubella Elimination, (RVC), cuyos miembros fueron nombrados por la Oficina Europea de la OMS. La primera actividad realizada por la Comisión Regional fue la elaboración de un marco estratégico para guiar el proceso de eliminación en la Región ${ }^{4}$. Desde la OMS se instó a los países a nombrar un Comité Nacional en cada estado miembro para la recogida, supervisión y envío de información sobre la situación del sarampión y la rubeola en un formulario estandarizado elaborado por la propia Comisión Regional.

El Comité Nacional debe realizar una revisión de las coberturas de vacunación sistemática y de la inmunidad de la población, la calidad de la vigilancia epidemiológica y las características de los virus aislados en los casos notificados. Esta información se incluye en un informe anual que se envía a la Comisión Regional junto con una declaración del Comité Nacional resumiendo la situación en el país.
Con la información recibida por todos los países, la Comisión Regional categoriza a los estados miembros en función de su situación de eliminación en base a unos criterios previamente establecidos, entre los que se encuentran la ausencia endémica de enfermedad, la existencia de un sistema de vigilancia de las enfermedades de alta calidad y la evidencia genotípica que apoye la interrupción de transmisión endémica ${ }^{4}$. El resultado se publica en un informe anual en el que se indican los países que de manera prioritaria deben establecer acciones aceleradas para mejorar la calidad de la información y mejorar algunas de las actividades específicas.

El Comité Nacional de Verificación de la Eliminación del Sarampión y la Rubeola en España es un comité independiente que se constituyó en marzo de 2013. Está formado por cinco miembros (tabla 1) que fueron nombrados por el Ministerio de Sanidad, Servicios Sociales e Igualdad. Las funciones de secretaría del Comité se ejercen desde el Área de Programas de Vacunación de la Subdirección General de Promoción de la Salud y Epidemiología del Ministerio de Sanidad. Actualmente se está elaborando un reglamento de funcionamiento del Comité.

Hasta el momento actual se han elaborado dos informes sobre la situación de España en cuanto a sarampión y rubeola, el primero sobre la situación en los años 2010 a 2012 y el segundo sobre la situación en el año 2013. En los informes anuales de 2013 y 2014 de la Comisión Regional se refleja la valoración de estos infor-

\begin{tabular}{|c|l|l|}
\hline \multicolumn{2}{|c|}{\begin{tabular}{c} 
Mabla 1 \\
\multicolumn{2}{|c|}{ Miembros del Comité Nacional de Verificación } \\
de la Eliminación del Sarampión y la Rubeola en España. Marzo de 2013
\end{tabular}} \\
\hline \multirow{4}{*}{ Presidente } & \multicolumn{1}{|c|}{ Nombre } & \multicolumn{1}{c|}{ Puesto de trabajo actual } \\
\hline \multirow{4}{*}{ Vocales } & D. Juan Fernando Martínez Navarro & Profesor Emérito en la Escuela Nacional de Sanidad. Madrid \\
\cline { 2 - 3 } & D. Angel Gil de Miguel & $\begin{array}{l}\text { Profesor de Medicina Preventiva en la Universidad Rey Juan } \\
\text { Carlos I. Madrid. }\end{array}$ \\
\cline { 2 - 3 } & D. Federico Martinón Torres & Pediatra en el Hospital de Santiago de Compostela. La Coruña. \\
\cline { 2 - 3 } & D. Francisco Salmerón García & $\begin{array}{l}\text { Jefe de División of Biotecnología y Productos Biotecnológicos } \\
\text { en la Agencia Española de Medicamentos y Productos Sanitarios. } \\
\text { Ministerio de Sanidad, Servicios Sociales e Igualdad. }\end{array}$ \\
\cline { 2 - 3 } & D. Rafael Fernández Muñoz & Experto en Virología. Investigador de proyecto del CSIC. \\
\hline
\end{tabular}


mes. En un artículo de la presente monografía se comenta la valoración del informe correspondiente al año $2013^{10}$. Recientemente, desde el Comité Nacional se ha enviado el informe referido a la situación en el año 2014, todavía pendiente de valorar por la Comisión Regional.

\section{PASOS PARA LA ACTUALIZACIÓN DEL PLAN}

Teniendo en cuenta las últimas actualizaciones realizadas por la OMS en las estrategias para alcanzar la eliminación y el establecimiento del proceso de verificación, se considera necesaria la actualización del Plan de eliminación de sarampión y rubeola en España.

Para ello se requiere, en primer lugar, la elaboración de un borrador de propuesta que deberá revisarse y discutirse en el seno de los órganos técnicos dependientes del Consejo Interterritorial, la Ponencia de Vigilancia Epidemiológica y la Ponencia de Programa y Registro de Vacunaciones. Además debe pasarse para su revisión al Comité Nacional de Verificación y a otras partes implicadas en el proceso de eliminación. La propuesta deberá elevarse para su aprobación por la Comisión de Salud Pública antes de su entrada en vigor.

En definitiva, es un procedimiento que requiere el acuerdo con todas las comunidades autónomas, tratando de obtener el máximo consenso posible para asegurar un trabajo coordinado y dirigido a la eliminación del sarampión y la rubeola en España.

\section{BIBLIOGRAFÍA}

1. Instituto de Salud Carlos III. Plan de eliminación del sarampión en España. Madrid: Instituto de Salud Carlos III; 2000. [citado el 22 agosto 2015]. Disponible en: http:// www.isciii.es/ISCIII/es/contenidos/fd-servicios-cientificotecnicos/fd-vigilancias-alertas/fd-enfermedades/fd-enfermedades-prevenibles-vacunacion/PLANSARAMPION. pdf.

2. WHO Regional Office for Europe. HEALTH 21: the health for all policy framework for the WHO European Region. Copenhagen: WHO Regional Office for Europe; 1999. (European Health for All Series, No. 6; [citado el 22 agosto 2015]. Disponible en: http://www.euro.who.int/ data/assets/pdf_file/0010/98398/wa540ga199heeng.pdf
3. WHO Regional Committee for Europe. Resolution EUR/ RC55/R7 on strengthening national immunization systems through measles and rubella elimination and prevention of congenital rubella infection in WHO's European Region. Copenhagen: WHO Regional Office for Europe; 2005. [citado el 22 agosto 2015]. Disponible en: http://www.euro.who. int/_data/assets/pdf_file/0003/88086/RC55_eres07.pdf

4. World Health Organization. Eliminating measles and rubella. Framework for the verificacion process in the WHO European Region. Copenhagen: World Health Organization. Regional Office for Europe; 2014. [citado el 23 de julio de 2015]. Disponible en: http://www.euro.who. int/_data/assets/pdf_file/0009/247356/Eliminating-measles-and-rubella-Framework-for-the-verification-processin-the-WHO-European-Region.pdf?ua $=1$

5. Instituto de Salud Carlos III. Protocolo de vigilancia de la rubeola y del síndrome de rubeola congénita en la fase de eliminación. Madrid: Instituto de Salud Carlos III; 2008. [citado el 22 agosto 2015]. Disponible en: http:// www.isciii.es/ISCIII/es/contenidos/fd-servicios-cientificotecnicos/fd-vigilancias-alertas/fd-enfermedades/fd-enfermedades-prevenibles-vacunacion/Protocoloeliminacionrubeola.pdf

6. Masa Calles J, Peña-Rey I, Castellanos Ruiz T, Martínez de Aragón MV. Protocolo de Vigilancia de la Rubéola y del Síndrome de Rubéola Congénita en la Fase de Eliminación. Bol Epidemiol Sem. 2010; 18:9-16. [citado el 22 agosto 2015]. Disponible en: http://revista.isciii.es/index. php/bes/article/view/2/1

7. WHO Regional Committee for Europe. Resolution EUR/RC60/R12 on renewed commitment to elimination of measles and rubella and prevention of congenital rubella syndrome by 2015 and sustained support for polio-free status in the WHO European Region. Copenhagen: WHO Regional Office for Europe; 2010. [citado el 22 agosto 2015]. Disponible en:http://www.euro.who.int/_data/assets/pdf_file/0016/122236/RC60_eRes12.pdf)

8. World Health Organization. Measles and rubella elimination 2015. Package for accelerated action: 20132015. Copenhagen: World Health Organization. Regional Office for Europe; 2013. [citado el 22 agosto 2015]. Disponible en: http://www.euro.who.int/ data/assets/ pdf_file/0020/215480/PACKAGE-FOR-ACCELERATED-ACTION-20132015.pdf?ua=1 [citado el 22 agosto 2015].

9. Regional Verification Commission for Measles and Rubella Elimination (RVC). World Health Organization. Regional Office for Europe. [citado el 22 agosto 2015]. Disponible en: Disponible en: http://www.euro.who.int/ en/health-topics/communicable-diseases/measles-andrubella/activities/regional-verification-commission-formeasles-and-rubella-elimination-rvc

10. Santos Preciado JI. Verifying the elimination of measles and rubella in the who european region: the case of spain. Rev Esp Salud Pública. 2015; 89(4):353-355. 